\title{
Properties of Distortion Risk Measures
}

\author{
Alejandro Balbás • José Garrido • Silvia Mayoral
}

\begin{abstract}
The current literature does not reach a consensus on which risk measures should be used in practice. Our objective is to give at least a partial solution to this problem. We study properties that a risk measure must satisfy to avoid inadequate portfolio selections. The properties that we propose for risk measures can help avoid the problems observed with popular measures, like Value at Risk $\left(V a R_{\alpha}\right)$ or Conditional $\operatorname{VaR}_{\alpha}\left(C V a R_{\alpha}\right)$. This leads to the definition of two new families: complete and adapted risk measures. Our focus is on risk measures generated by distortion functions. Two new properties are put forward for these: completeness, ensuring that the distortion risk measure uses all the information of the loss distribution, and adaptability, forcing the measure to use this information adequately.
\end{abstract}

Keywords Risk measures - Distortion functions $\cdot \mathrm{VaR}_{\alpha} \cdot \mathrm{CVa} R_{\alpha}$. Coherent measures $\cdot$ Complete measures $\cdot$ Adapted measures

AMS 2000 Subject Classification 62P05 • 91B28

This research was partially funded by ${ }^{1,3}$ Welzia Management, SGIIC SA, RD Sistemas SA, Comunidad Autónoma de Madrid Grant s-0505/tic/000230, and $M E y C$ Grant BEC2000-1388-C04-03 and by ${ }^{2}$ the Natural Sciences and Engineering Research Council of Canada (NSERC) Grant 36860-06.

\footnotetext{
A. Balbás

Universidad Carlos III de Madrid, Madrid, Spain

e-mail: balbas@emp.uc3m.es

J. Garrido (凶)

Concordia University, Montreal, Canada

e-mail: garrido@mathstat.concordia.ca

S. Mayoral

Universidad de Navarra, Pamplona, Spain

e-mail: smayoral@unav.es
} 


\section{Introduction}

For some time Value at Risk $\left(V_{a} R_{\alpha}\right)$ was the preferred choice in industry to measure market risk. The main advantage of $V a R_{\alpha}$, over other risk measures is that when applied to any financial instrument, it is always expressed as money losses. In addition, $V a R_{\alpha}$ is simple to use with a wide variety of risks. Despite its universality, several authors have pointed out the deficiencies of $\mathrm{VaR}$, such as its lack of subadditivity (Artzner et al. 1997, 1999) or of convexity, and that it is a measure difficult to optimize as it may have multiple local minima (Basak and Shapiro 2001).

Artzner et al. (1997) address the question of how a risk measure should behave for different risks on a finite-states probability space. The authors suggest a set of properties for a risk measure to be coherent: sub-additivity, translation invariance, positive homogeneity and monotonicity. Their study was extended to general probability spaces in Delbaen (2002).

With the concept of coherent risk measure and its defining properties, different sets of measures started to appear, each with distinctive properties: convex measures (see Föllmer and Shied 2002 or Fritelli and Rosazza 2002), spectral measures (Acerbi 2002) or deviation measures (Rockafellar et al. 2006a). Denuit et al. (2006) divide the axioms to characterize a risk measures into three classes: rationality, additivity and technical axioms.

The above risk measures are obtained axiomatically from their desired properties. But risk measures can be generated in other ways. For instance, Denuit et al. (2006) determine the functional form of their risk measures through economic indifference arguments, such as expected utility and distortion utility. Goovaerts et al. (2003b) recover many existing risk measures by minimizing a Markov bound for the tail probability.

Applications of the risk measures to portfolio optimization can be found in Rockafellar and Uryasev (2000) for $C V a R_{\alpha}$, Ruszczynski and Shapiro (2006) for convex risk measures, Acerbi and Simonetti (2002) for spectral risk measures and Rockafellar et al. (2006b) for deviation risk measures.

Our objective is to show that some of these risk measures do not satisfy all the properties required to avoid inconsistent decisions. We first define a completeness property that all measures must have. Then, depending on the intended use of the risk measure, we impose a second set of properties: exhaustivity and adaptability.

The paper is organized as follows. Section 2 reviews the properties that the literature proposes for risk measures, highlighting differences between existing families of risk measures and singling out the most popular measures in practice.

Section 3 studies distortion risk measures and their properties. Then Section 4 gives some examples of distortion risk measures. It shows how some popular measures, such as Conditional Value at Risk $\left(C V a R_{\alpha}\right)$, lead to inconsistent decisions. To avoid this problem, the completeness property is put forward. It ensures that the distortion risk measure uses all the information in the original loss distribution. It shows also how inconsistencies are due to the constant portions of the distortion function generating the risk measure, rather than its non-differentiability, as suggested by Wang (2002). We characterize this property through the monotonicity of the distortion function with a condition that is easily verified.

Section 5 shows that completeness is not sufficient, in general, for the distortion risk measure to avoid inconsistencies. An adaptability property is defined, forcing 
the risk measure to use the information in the original loss distribution adequately. This property is characterized through simple conditions on the derivative of the distortion function.

The conclusion gives a table summarizing the different properties that common distortion risk measures satisfy.

\section{Properties of Risk Measures}

Let $\Omega$ be a sample space and $X: \Omega \rightarrow \mathbb{R}$ be the loss or gain variable (risk) associated with a given investment, over a single period of time 0 to $T$. Then consider a probability space $(\Omega, \mathbb{P})$ and let $\mathbb{X}$ be the set of all risks, that is, all real functions on $\Omega$. A risk measure is defined as follows.

Definition 2.1 A risk measure is a function $\rho: \mathbb{X} \rightarrow \mathbb{R}$.

If the value $\rho(X)$, assigned by the measure $\rho$ to risk $X$ is positive, then it can be interpreted as the minimum amount of money that an agent must add to the position $X$, by investing at the risk free rate, to forego any level of risk. By contrast, if $\rho(X)$ is negative, then the amount $-\rho(X)$ can be cashed, without risk, from the current position.

According to Duffie and Pan (1997), $V a R_{\alpha}$ can be defined as (for the market value of an asset, it could be defined in more general contexts):

For a given time horizon $T$ and an $\alpha \times 100 \%$ confidence level, $V a R_{\alpha}$ is the loss in market value that can only be exceeded with a probability of at most $1-\alpha$.

$\operatorname{VaR} R_{\alpha}$ usually answers the question: What is the minimal loss incurred in the $(1-$ $\alpha) \times 100 \%$ worse outcomes of the portfolios?

Actually, $\operatorname{VaR}_{\alpha}$ is simply the $\alpha \times 100 \%$-percentile of the loss distribution.

Definition 2.2 For a risk $X$ over a given period $[0, T]$ and $0<\alpha<1$, the $\alpha \times 100 \%$ Value at Risk is:

$$
\operatorname{VaR}_{\alpha}(X)=\sup \{x \in \mathbb{R} \mid \mathbb{P}(X \geq x)>1-\alpha\} .
$$

One main advantage of $V a R_{\alpha}$ is that, irrespectively of the financial instrument to which it is applied, it is always expressed as money lost. In addition it is simple to calculate for a wide variety of risks. But despite its universality, several authors have pointed out the deficiencies of $V a R_{\alpha}$ : it is not sub-additive, nor convex and difficult to optimize.

Artzner et al. (1997) consider how risk measures should behave. They suggest a set of properties that coherent risk measure should satisfy.

Definition 2.3 (Artzner et al. 1997) A risk measure $\rho$ is called coherent if and only if it satisfies the following axioms:

1. Sub-additivity: for any $X, Y \in \mathbb{X}$, then $\rho(X+Y) \leq \rho(X)+\rho(Y)$,

2. Positive homogeneity: for any $X \in \mathbb{X}$ and $\lambda \geq 0$, then $\rho(\lambda X)=\lambda \rho(X)$. 
3. Translation invariance: for a fixed $X \in \mathbb{X}$ and any $a \in \mathbb{R}$, then $\rho(X+a)=$ $\rho(X)+a$.

4. Monotonicity: let $X, Y \in \mathbb{X}$ be such that $X \leq Y$ a.s., then $\rho(X) \leq \rho(Y)$.

Artzner et al. (1999) show that such measures are characterized in terms of scenarios. In fact, the choice of a risk measure becomes equivalent to the choice of a set of generalized scenarios (see Song and Yan 2006, for more general characterizations).

For losses, an alternative to $V a R_{\alpha}$ is the so-called Conditional Value at Risk $\left(C V a R_{\alpha}\right)$, also known as Conditional Tail Expectation $(C T E)$, although this is sometimes a misnomer. It was initially proposed by Artzner et al. (1999) and has been studied extensively in recent years.

$C V a R_{\alpha}$ answers the question: What is the expected loss incurred in the $(1-\alpha) \times$ $100 \%$ worse cases in closing a position?

Definition 2.4 (Artzner et al. 1999) For a risk $X$ on $(\Omega, \mathbb{P})$ and a confidence level $0<\alpha<1$, the $\alpha \times 100 \% C V a R_{\alpha}$ is:

$$
\operatorname{CVaR}_{\alpha}(X)=\frac{1}{1-\alpha} \int_{0}^{1-\alpha} \operatorname{VaR}_{t}(X) d t .
$$

By definition, $C V a R_{\alpha}(X) \geq \operatorname{VaR}_{\alpha}(X)$, for any risk $X$. The properties of $C V a R_{\alpha}$ are more attractive than those of $V a R_{\alpha}$ : sub-additive and convex. In fact, it is in most cases coherent (Artzner et al. 1999).

Föllmer and Shied (2002) suggest that market risk may increase non-linearly with the value of the position. For example, it is possible that a liquidity risk be created when a position is multiplied by a sufficiently large factor. Accordingly, they propose relaxing the conditions of positive homogeneity and sub-additivity to convexity (see also Fritelli and Rosazza 2002).

Definition 2.5 (Föllmer and Shied 2002) A a risk measure $\rho$ is called convex if and only if $\rho[\lambda X+(1-\lambda) Y] \leq \lambda \rho(X)+(1-\lambda) \rho(Y)$, for any risk $X$ on $(\Omega, \mathbb{P})$ and weight $\lambda \in[0,1]$.

Convexity implies that diversification does not increase risk, as the risk value of the diversified portfolio $\lambda X+(1-\lambda) Y$ is less or equal to the re-weighted average of the individual risk values.

Föllmer and Schied also show that, as with coherent risk measures, convex risk measures can be represented in terms of a set of scenarios.

Acerbi (2002) defines spectral measures. They are also studied with a different approach by Kusuoka (2001), who shows that a spectral measure can be associated to coherent risk measures that have two additional properties, law invariance and comonotone additivity (see Denneberg 1994 for the latter). Law invariance is important in practice as it is required for the estimation of the risk measure from empirical data. Coherent risk measures that are not law invariant (for example, worst conditional expectation, $W C E_{\alpha}$, of Artzner et al. 1999) cannot be estimated solely from data. Using such measures can lead to different risk values for two portfolios with identical loss distributions. 
Rockafellar et al. (2006a) propose two different sets of risk measures: deviation measures and measures bounded in expectation. Although the former are used in practice, they are not coherent. While the latter are related to deviation measures, but do satisfy some axioms of Artzner et al. (1997), replacing the monotonicity property by one called boundedness in expectation.

Clearly, opinions differ substantially on the properties that risk measures should satisfy. Goovaerts et al. (2003a) are critical of some conditions for coherence; subadditivity, translation invariance and positive homogeneity. Dhaene et al. (2008) argue that the property of subadditivity can lead to undesirable situations, for example an expected shortfall that increases with a merger.

Desirable properties differ with the intended use for a risk measure: capital requirements, statutory purposes, or risk premium calculations. We may conclude that there is no general set of axioms that is valid for all above applications.

The following section studies the relevance of certain properties when restricting attention to distortion risk measures. Several simple examples serve to illustrate the inconsistencies obtained with some of the most popular risk measures. We discuss different properties that distortion risk measures should satisfy to avoid such problems. These properties find simple interpretations in terms of the distortion function $g$ that characterizes the risk measure, and its derivative $g^{\prime}$.

\section{Distortion Risk Measures}

Consider the set function $\mu: \mathcal{F} \rightarrow[0, \infty)$, defined on the $\sigma$-algebra $\mathcal{F}$, such that $\mu(\emptyset)=0$ and $A \subseteq B \Rightarrow \mu(A) \leq \mu(B)$, for $A, B \in \mathcal{F}$.

If

$$
\mu(A \cup B) \leq \mu(A)+\mu(B), \quad \text { for all } A, B \in \mathcal{F},
$$

$\mu$ is called sub-additive.

Definition 3.1 A distortion function $g:[0,1] \rightarrow[0,1]$ is a non-decreasing function such that $g(0)=0$ and $g(1)=1$. Furthermore, the dual distortion function of $g$ is given by:

$$
\tilde{g}(u)=1-g(1-u), \quad u \in[0,1] .
$$

We consider the special case $\mu(A)=g[P(X \in A)]:=P^{*}(A)$, where $g$ is a distortion function, $P$ is a probability measure on the $\sigma$-algebra $\mathcal{B}$ of Borel on $\Re$, and $X$ is a random variable. Such a function $\mu$ is called a distorted probability $P^{*}{ }^{1}$ Similarly, consider its dual function $\tilde{\mu}(A)=\widetilde{g}[P(X \in A)]:=\widetilde{P}^{*}(A)$.

We study risk measures for financial and actuarial instruments defined through the Choquet integral of the random variable for the portfolio gains and losses:

$$
\rho_{g}[X]=\int X d P^{*}=\int X^{+} d P^{*}-\int X^{-} d \widetilde{P}^{*},
$$

if these integrals are finite (Denneberg 1994).

\footnotetext{
${ }^{1}$ Note that $P^{*}$ is not really a probability measure, but it is usually referred to as a "distorted probability" in the literature.
} 
Consider this special case of risk measures based on a distorted probability. It can be shown (Wang 1996) that the Choquet integral of a random variable $X$, with respect to a distortion function $g$, is equivalent to the expectation of the variable $X$ under the distorted probability $P^{*}$ (with corresponding distribution $F^{*}$ and survival function $\left.S^{*}=1-F^{*}\right)$ :

$$
\begin{aligned}
\mathbb{E}_{P^{*}}(X) & =\int_{0}^{\infty} S^{*}(x) d x-\int_{0}^{\infty} F^{*}(-x) d x \\
& =\int_{0}^{\infty} g[S(x)] d x-\int_{0}^{\infty} \tilde{g}[F(-x)] d x \\
& =\int_{0}^{\infty} g[S(x)] d x-\int_{0}^{\infty}\{1-g[S(-x)]\} d x=\rho_{g}[X]
\end{aligned}
$$

Such a distortion risk measure is then the mean of a new variable where the probabilities have been re-weighted.

By construction, it can easily be shown that distortion risk measures satisfy several properties in Definition 2.3. That is, by the properties of Choquet integrals and independently of the choice distortion function, these are translation invariant, positively homogeneous, monotone and comonotonely additive. In addition, it has been shown for positive losses that a distortion risk measure is coherent if and only if the distortion function is concave (Wirch and Hardy 2001). Finally, it can be shown that if $g$ is concave, the resulting distortion risk measure is spectral. A relationship between coherent and spectral risk measures can be found in Gzyl and Mayoral (2008).

Depending on the chosen distortion function, different risk measures are obtained. Special cases are $V a R_{\alpha}, C V a R_{\alpha}$ and the $W T$ measure of Wang (2000).

The $V a R_{\alpha}$ risk measure only uses information on the frequency of losses, not their severity. For instance, doubling the size of the maximal loss has no influence on $\operatorname{VaR}$.

To study this further, let us express $V a R_{\alpha}$ as a Choquet integral with respect to a distorted probability. Here the needed distortion function is (see Fig. 1):

$$
g(u)=\left\{\begin{array}{ll}
0 & \text { if } 0 \leq u<1-\alpha \\
1 & \text { if } 1-\alpha \leq u \leq 1
\end{array},\right.
$$

and then $V a R_{\alpha}$ is simply given by:

$$
\operatorname{VaR}_{\alpha}=\int_{0}^{\infty} g\left[S_{X^{+}}(x)\right] d x-\int_{0}^{\infty} \tilde{g}\left[S_{X^{-}}(x)\right] d x=\int_{0}^{x_{\alpha}} d x=x_{\alpha}
$$

where $x_{\alpha}$ is the $\alpha \times 100 \%$-percentile of the distribution of $X$.

From Fig. 1 we see that $V a R_{\alpha}$ 's distortion $g$ is non-decreasing, with $g(0)=0$, $g(1)=1$, piece-wise constant but not concave. Hence the risk measure associated with this distortion function is not coherent. 
Fig. 1 Distortion functions

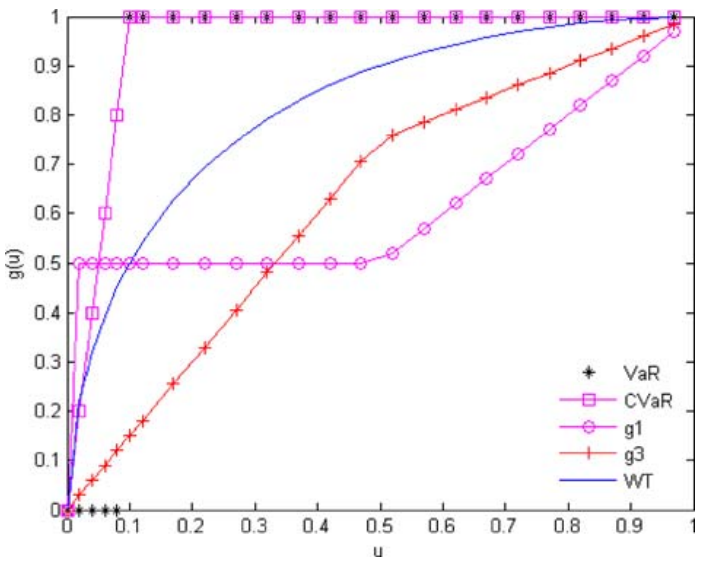

By contrast, the $C V a R_{\alpha}$ risk measure uses both, the frequency and the expected severity of the loss in excess of $V a R_{\alpha}$. This measure can also be expressed as a Choquet integral with respect to a distorted probability (see Fig. 1):

$$
g(u)=\left\{\begin{array}{ll}
\frac{u}{1-\alpha} & \text { if } 0 \leq u \leq 1-\alpha \\
1 & \text { if } 1-\alpha \leq u \leq 1
\end{array} .\right.
$$

Here $g$ is also a non-decreasing distortion function, which is continuous and concave, but not differentiable. Since $g$ is concave, $C V a R_{\alpha}$ is spectral and coherent. A general overview of distortion risk measures and their relation with orderings of risks and the concept of comonotonicity can be found in Dhaene et al. (2006).

\section{Properties of Distortion Risk Measures}

\subsection{Complete Distortion Risk Measures}

One problem with $C V a R_{\alpha}$ is that it only uses the severity of losses in excess of $V a R_{\alpha}$, disregarding smaller losses, those less than the $\alpha \times 100 \%$-percentile. In some cases this can lead to erroneous decisions, as illustrated with the simple example that follows. In addition, this measure is not robust to extreme losses.

Example 4.1 Let $\mathrm{A}$ and $\mathrm{B}$ be 2 portfolios with the loss probabilities $P_{A}$ and $P_{B}$ given in Table 1:

Table 1 Original and $C V a R_{\alpha}$-distorted loss probabilities for Portfolios A and B

\begin{tabular}{lllll}
\hline Loss & $P_{A}(x)$ & $P_{A}^{*}(x)$ & $P_{B}(x)$ & $P_{B}^{*}(x)$ \\
\hline 0 & 0.600 & 0 & - & - \\
9 & - & - & 0.600 & 0 \\
10 & 0.400 & 1 & 0.400 & 1 \\
\hline
\end{tabular}


For these losses Table 1 gives the distorted probabilities $P_{A}^{*}$ and $P_{B}^{*}$ based on $g$ in Eq. 4 with $\alpha=0.95$.

Here the expected excess loss is identical in both portfolios. Using Eq. 2 we see that also $C V a R_{0.95}=10$ for both portfolios. Still, the loss is positive with certainty in Portfolio B. In fact, Portfolio B dominates stochastically Portfolio A. Hence the risk measure should detect that Portfolio B is more risky.

Wang (2002) conjectures that the problem with the $C V a R_{\alpha}$ distortion function is that it assigns a 0 -value to all percentiles below the $\alpha$ significance level. This may lead to think that restricting the distortion function $g$ to be strictly positive after the origin may solve the problem. The following simple example, similar to Example 4.1, defines a distortion function that only takes value 0 at the origin, $g(0)=0$, yet the resulting risk measure still exhibits inconsistency problems.

Example 4.2 Consider the following distortion function (see Fig. 1):

$$
g_{1}(u)= \begin{cases}50 u & \text { if } 0 \leq u<0.01 \\ 0.5 & \text { if } 0.01 \leq u<0.5 \\ u & \text { if } 0.5<u \leq 1\end{cases}
$$

It is continuous, not differentiable at $u=0.01$ and $u=0.5$ and constant over the interval $[0.01,0.5]$. Consider again two simple portfolios, both with a maximum loss of 11 but different medium losses, 1 in the case of Portfolio A and 10 for Portfolio B (Table 2).

Here the distortion risk measure generated by $g_{1}$ is equal to 5.5 , for both portfolios. Still, Portfolio A is clearly more risky than B, with it losses larger or equal to 10 with a probability of 0.4 .

The problem does not lie in the differentiability of the distortion function, as conjectured in Wang (2002), but rather that $g_{1}$ is constant on an interval, losing information on the original loss distribution. Calculating the distorted probabilities, we see that these are 0 for the intermediate losses of both portfolios, doing away with the distinct probabilities at 1 and 10 if the original loss distributions.

It is natural to ask if rather than its differentiability, the problem could be rooted in the convexity of the distortion function. The answer is no, since replacing $g_{1}$ by another convex distortion function such as:

$$
g_{2}(u)= \begin{cases}\frac{1}{3} u & \text { if } 0 \leq u<\frac{1}{3} \\ \frac{4}{3} u-\frac{1}{3} & \text { if } \frac{1}{3} \leq u \leq 1\end{cases}
$$

Table 2 Original and $g_{1}$-distorted loss probabilities for Portfolios A and B

\begin{tabular}{lllll}
\hline Loss & $P_{A}(x)$ & $P_{A}^{*}(x)$ & $P_{B}(x)$ & $P_{B}^{*}(x)$ \\
\hline 0 & 0.600 & 0.500 & 0.600 & 0.500 \\
1 & - & - & 0.390 & 0 \\
10 & 0.375 & 0 & - & - \\
11 & 0.025 & 0.500 & 0.010 & 0.500 \\
\hline
\end{tabular}


yields a higher distortion risk measure for Portfolio A (of about 2) than for B (about $0,23)$. The key difference here is that $g_{2}$ is not constant on an interval, irrespective of its convexity or concavity. As such $g_{2}$ uses all the information in the original loss distribution. This is explained more precisely by the concept of completeness, defined as follows.

Definition 4.1 Let $X$ be a random variable and $\rho_{g}$ a distortion risk measure generated by $\rho_{g}(X)=\mathbb{E}_{P^{*}}(X)$ as in Eq. 2 . We say that $\rho_{g}$ is a complete distortion risk measure if:

$$
S\left(x_{1}\right)=S\left(x_{2}\right) \quad \Leftrightarrow \quad S^{*}\left(x_{1}\right)=S^{*}\left(x_{2}\right), \quad \forall x_{1}, x_{2} \in[0, \infty),
$$

where $S^{*}$ is the survival function of the distorted distribution.

Note that the above definition could be expressed, equivalently, in terms of the probability functions $P$ and $P^{*}$.

It is easily seen that $V a R_{\alpha}$ and $C V a R_{\alpha}$ are not complete risk measures, as the distortion function of $V a R_{\alpha}$ is constant on all its domain, while that of $C V a R_{\alpha}$ is constant on the interval $[1-\alpha, \infty)$. This explains, in part, the inconsistency problems sometimes produced by $C V a R_{\alpha}$.

An example of a complete distortion risk measures is Wang's Transform (WT, see Wang 2000). He chooses a parametric family of symmetric functions around 0 , so that the transform preserves certain properties of the original loss distribution. WT is defined as (see Fig. 1):

$$
g_{\lambda}(u)=\Phi\left[\Phi^{-1}(u)+\lambda\right], \quad u \in[0,1],
$$

where $\Phi$ is the distribution of a standard normal. The parameter $\lambda$ is called the market price of risk and reflects systematic risk. It was coined WT by financial engineers and was inspired by three important papers: Venter $(1991,1998)$ and Butsic (1999).

Complete distortion risk measures are simply characterized. It is sufficient for their corresponding distortion function to be strictly increasing. This property was also pointed out in the unpublished work by Wirch and Hardy (2000) who prove that any distortion risk measures derived from a distortion function that is concave, but no strictly concave, does not strongly preserve stop-loss order.

Theorem 4.1 Let $P^{*}$ be a distorted probability defined by the distortion function $g$ in Eq. 2. The following conditions are equivalent:

1. $\rho_{g}$ is complete: i.e. $S\left(x_{1}\right)=S\left(x_{2}\right) \Leftrightarrow S^{*}\left(x_{1}\right)=S^{*}\left(x_{2}\right)$, for all $x_{1}, x_{2} \in[0, \infty)$,

2. $g$ is strictly increasing.

Proof The distorted survival function of $X$ is given by $S^{*}(x)=g(S(x))$.

Assume Condition 1. Since $g$ is a distortion function, by definition it is nondecreasing. Hence to see that it is strictly increasing is equivalent to check that

$$
u=v \quad \Leftrightarrow \quad g(u)=g(v), \quad u, v \in[0,1],
$$

which is Condition 1. 
Alternatively, if $g$ is strictly increasing then Eq. 8 holds. Taking $u=S\left(x_{1}\right)$ and $v=S\left(x_{2}\right)$ yields Condition 1 .

Note that the distortion function in Eq. 5 is strictly increasing, while the distortion function generated by $C V a R_{\alpha}$, in Eq. 4, is not.

\subsection{Exhaustive Distortion Risk Measures}

A key difference between using a convex or a concave distortion function is that the resulting risk measure is super-additive or sub-additive, respectively. Hence, it is not only necessary for a risk measure to be sub-additive (resp. super-additive), but also that it uses all the information of the initial loss distribution, that is that it be complete. As such, when interested in sub-additive risk measures (which is synonymous to coherence in the case of distortion risk measures) we define a new set of exhaustive risk measures, as those being coherent and complete.

Definition 4.2 Let $X$ be a random variable and $\rho_{g}$ a distortion risk measure generated by $\rho_{g}(X)=\mathbb{E}_{P^{*}}(X)$. We say that $\rho_{g}$ is an exhaustive distortion risk measure if it is coherent and complete.

From Theorem 4.1 it follows that distortion risk measures are exhaustive if their distortion function $g$ is concave and strictly increasing.

Corollary 4.1 Let $\rho_{g}$ be the distortion risk measure generated by $g$. Then $\rho_{g}$ is exhaustive if and only if $g$ is concave and strictly increasing.

It follows from the above corollary that a sufficient and necessary condition that characterizes exhaustive distortion risk measures is that $g$ be 1 at and only at $x=1$.

Corollary 4.2 Let $\rho_{g}$ be the distortion risk measure generated by $g$. It is exhaustive if and only if $g$ is concave and $g(x)<1 \forall x<1$.

Proof By Corollary 4.1 it is sufficient to show that $g$ is concave and strictly increasing.

$(\Leftarrow)$ : If $g$ is strictly increasing it cannot be constant on an interval. Hence $\nexists \epsilon>0$ such that $g(1-\epsilon)=1 \Rightarrow g\left(1^{-}\right)<1$.

$(\Rightarrow)$ : Let $g$ be concave with $g\left(1^{-}\right)<1$. We need to show that $g$ is strictly increasing. If $g$ is constant on an interval $[x, y]$, that is $\exists x, y$ such that $x<y$ and $g(x)=g(y)$, then $x<y<1$ as it must satisfy $g\left(1^{-}\right)<1$. Now since distortion functions are defined to be increasing, then $g(x)=g(y)<g(1)$. Take $\lambda>0$ such that $\lambda x+(1-\lambda) 1=y$. Applying $g$ to both sides of the equality gives $g[\lambda x+(1-\lambda) 1]=g(y)$. But again by definition $g$ is increasing with $g(1)=1$, implying that:

$$
g[\lambda x+(1-\lambda)]=g(y)=g(x) \leq \lambda g(x)+(1-\lambda) .
$$

This contradicts the assumption that $g$ be concave.

This last property does not apply to distortion risk measures generated by convex functions $g$, that is for super-additive and complete distortion risk measures. For 
example the distortion function in Eq. 5 is such that $g\left(1^{-}\right)<1$ but does not generate a complete distortion risk measure.

\section{Discussion}

The previous section defines complete distortion risk measures to ensure that these use all the information in the original loss distribution. A natural question is to ask if completeness is sufficient to avoid the type of inconsistencies illustrated by Example 4.1. This section shows that the answer is no and that additional conditions need to be imposed on the distortion function.

Example 5.1 Consider the following distortion function (see Fig. 1):

$$
g_{3}(u)=\left\{\begin{array}{ll}
\frac{3}{2} u & \text { if } 0 \leq u<\frac{1}{2} \\
\frac{1}{2} u+\frac{1}{2} & \text { if } \frac{1}{2} \leq u \leq 1
\end{array},\right.
$$

and the following loss distributions for 2 distinct portfolios (Table 3 ).

Here $g_{3}$ is concave and satisfies Eq. 6, hence its corresponding distortion risk measure is exhaustive. Still, it produces the same risk measure value of $21 / 8=2.625$ for both portfolios, while Portfolio A is clearly more risky than B, with its larger maximal loss.

This type of inconsistency is not due to the non-differentiability of the distortion function in Eq. 9, but rather to its piece-wise linearity. This results in a distortion that re-weighs different probabilities equally. All values $0 \leq S_{X}(x)<\frac{1}{2}$ in Example 5.1 are re-weighted by a multiplicative factor of $\frac{3}{2}$, while with values starting at $S_{X}(x)=$ $\frac{1}{2}$ the re-weighting factor becomes $\frac{1}{2}$, independently of the value of $S_{X}(x)$, that is the original loss frequency and severity. Note that $V a R_{\alpha}$ and $C V a R_{\alpha}$ are two other examples of piece-wise linear distortion functions.

A simple illustration, like Example 5.1, but for convex piece-wise linear distortion functions would show the same inconsistencies due to the constant slopes and their resulting constant re-weighting factors.

Since the slope gives the re-weighting factor of a (piece-wise) linear function like that in Eq. 9, then we propose to measure the re-weighting factor for a general distortion $g$ at a point $x$ as the slope $g^{\prime}(x)$ of the tangent to $g$ at $x$.

It can be shown that distortion functions that lead to coherent risk measures apply a re-weighting factor larger than 1 to large losses, hence assigning them a distorted probability greater than the initial one. By contrast, small losses are assigned smaller probabilities than by the initial distribution.

Table 3 Original and $g_{3}$-distorted loss probabilities for Portfolios A and B

\begin{tabular}{lllll}
\hline Loss & $P_{A}(x)$ & $P_{A}^{*}(x)$ & $P_{B}(x)$ & $P_{B}^{*}(x)$ \\
\hline 0 & 0.500 & 0.250 & 0.450 & 0.225 \\
1.8 & - & - & 0.350 & 0.475 \\
2 & 0.375 & 0.5625 & - & - \\
5.9 & - & - & 0.200 & 0.300 \\
8 & 0.125 & 0.1875 & - & - \\
\hline
\end{tabular}


Fig. 2 Distorted normal $(0,1)$ survival functions

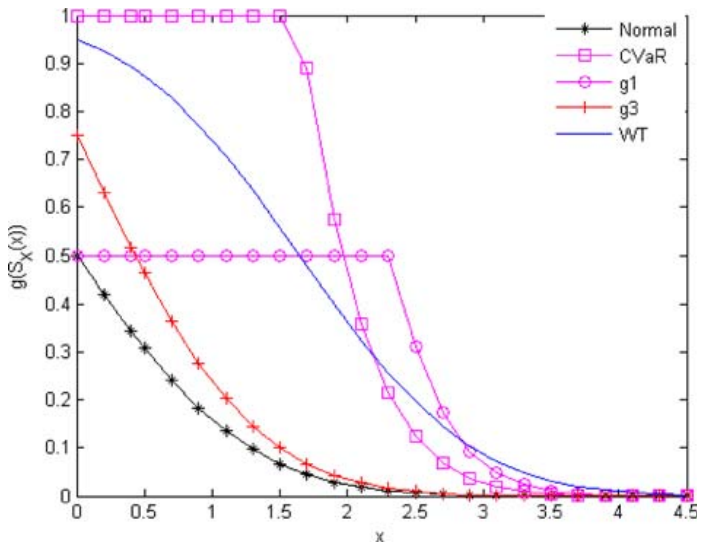

Similarly, it can be shown that for convex risk measures the opposite is true: the re-weighting factor is smaller than 1 for large losses and larger than 1 for large ones.

Figure 2 shows how a standard normal initial loss function (lowest curve) is transformed by the different distortion functions discussed above. We can see how concave distortions emphasize large losses, thickening the right tail of the distorted distributions. See also how $C V a R_{\alpha}$ and $g_{1}$ assign 0-probabilities (i.e. their distorted survival functions $g\left[S_{X}(x)\right]$ are constant) over sub-intervals. This means they do not use the information in parts of the original loss distribution.

The following result links this discussion about re-weighting factors and the concept of exhaustive risk measures. It also revisits the idea of differentiability of Wang for distortion functions.

Theorem 5.1 Let $g$ be a concave (convex) distortion function, such that $g(u) \neq u$ for any $u \in(0,1)$. Then $\exists d \in(0,1)$ such that $g^{\prime}(u) \geq(\leq) 1$, for all $u \in[0, d]$ and $g^{\prime}(u) \leq$ $(\geq) 1$, for all $u \in[d, 1]$.

Proof $g$ being a concave distortion function, $g(u) \neq u$ implies that $g(u)>u$, for all $u \in(0,1)$. Hence

$$
g^{\prime}\left(0^{+}\right)=\lim _{h \rightarrow 0^{+}} \frac{g(0+h)-g(0)}{h}=\lim _{h \rightarrow 0^{+}} \frac{g(h)-0}{h}>\lim _{h \rightarrow 0^{+}} \frac{h-0}{h}=1
$$

and

$$
\begin{aligned}
g^{\prime}\left(1^{-}\right) & =\lim _{h \rightarrow 0^{-}} \frac{g(1+h)-g(1)}{h}=\lim _{h \rightarrow 0^{-}} \frac{g(1+h)-1}{h} \\
& =\lim _{h \rightarrow 0^{+}} \frac{g(1-h)-1}{-h}=\lim _{h \rightarrow 0^{+}} \frac{1-g(1-h)}{h}<\frac{1-(1-h)}{h}=1 .
\end{aligned}
$$

Again by concavity, necessarily $g$ must be continuous on the interval $[0,1]$ hence there must exist $a \in(0,1)$ such that $g^{\prime}(u)>1$ for all $u \in[0, a]$ and $b \in(0,1)$ such that $g^{\prime}(u)<1$ for all $u \in[d, 1]$. Also by its concavity, $g$ has a non-increasing derivative, hence there must exist $d \in(a, b)$ such that $g^{\prime}(d)=1$, which completes the proof. 
Remark 5.1 Since $g$ is required only to be concave, then $d$ in Theorem 5.1 may not be unique. To ensure uniqueness $g$ needs to be strictly concave.

In other words, Theorem 5.1 implies that the investor re-weighs by a factor larger than 1 over the interval $(0, d)$, that is for extreme losses $\left\{x \in[0, \infty): S_{X}(x)<d\right\}$. This distorts the tail of the loss distribution into a thicker tail. By contrast, the reweighting factor is smaller than 1 for $\left\{x \in[0, \infty): S_{X}(x)>d\right\}$.

In conclusion, a coherent distorted risk measure is the expected value under a new loss distribution with a thicker right-tail than the original one. This is reasonable for an investor who wants to protect against extreme losses, by emphasizing them more than in the original loss distribution, while de-emphasizing smaller losses.

For instance, in Example 5.1, the investor gives a larger weight to losses that have a survival probability less than $\frac{1}{2}$, as the slope of the distortion function jumps from $\frac{3}{2}$ to $\frac{1}{2}$ at the point $d=\frac{1}{2}$.

The natural question is at what change-point should the re-weighting factor become larger than 1? Under the notation of Theorem 5.1 this reduces to the choice of $d$, such that the distorted $S^{*}(x)>S(x)$ whenever $S(x)<d$, but $S^{*}(x)<S(x)$ when $S(x)>d$.

For $V_{a} R_{\alpha}$ and $C V a R_{\alpha}$, this re-weighting change-point is precisely the $\alpha$ percentile. In the case of $C V a R_{\alpha}$, the weight given to values larger than the $\alpha$ percentile is maximal (largest possible of $g$ while satisfying $g(0)=0$ ), but is minimal (i.e. 0 ) for values smaller than the $\alpha$-percentile.

In light of the inconsistency problems illustrated by Example 5.1 and the need to protect against extreme losses, we propose a final property for distortion risk measures that preserves diversification (i.e. concavity).

Definition 5.1 A distortion risk measure is called adapted if its distortion function:

1. $g$ is strictly concave, that is $g^{\prime}$ is strictly decreasing.

2. $\lim _{u \rightarrow 0^{+}} g^{\prime}(u)=\infty$ and $\lim _{u \rightarrow 1^{-}} g^{\prime}(u)=0$.

The condition $g^{\prime}(0)=\infty$ is needed to ensure unbounded relative loadings for losses in the extreme right tail (see Wang 1996).

Adapted risk measures re-weigh different losses differently, giving the smallest distorted probability to losses near 0 while large losses are assigned maximal probabilities.

It is easily shown that adapted risk measures are exhaustive, but the converse is not true.

$C V a R_{\alpha}$ is not an adapted risk measure since $g^{\prime}(u)=0$ is for $u \in[1-\alpha, 1]$ and constant $g^{\prime}(u)=\frac{1}{1-\alpha}$ for $u \in[0,1-\alpha]$. The distortion risk measure generated by $g_{3}$ in Eq. 9 is not adapted either, as its derivative is also constant, $g_{3}^{\prime}(u)=\frac{3}{2}$ for $u \in\left[0, \frac{1}{2}\right]$, while $g_{3}^{\prime}(u)=\frac{1}{2}$ for $u \in\left[\frac{1}{2}, 1\right]$.

Other examples of distortion functions are the dual-power $g_{D P}$ and the proportional hazard transform $g_{P H}$ functions:

$$
\begin{aligned}
& g_{D P}(u)=1-(1-u)^{v}, \quad u \in[0,1], \quad v \geq 1 \\
& g_{P H}(u)=u^{\frac{1}{\gamma}}, \quad u \in[0,1], \quad \gamma \geq 1 .
\end{aligned}
$$


Table 4 Properties of the distortion risk measures

\begin{tabular}{llllll}
\hline Measure & Coherent & Complete & Exhaustive & Adaptable & Condition 2 \\
\hline$V a R_{\alpha}$ & No & No & No & No & No \\
$C V a R_{\alpha}$ & Yes & No & No & No & No \\
$g_{3}$ & Yes & Yes & Yes & No & No \\
$D P(v>1)$ & Yes & Yes & Yes & No & Yes \\
$P H(\gamma>1)$ & Yes & Yes & Yes & No & Yes \\
$W T$ & Yes & Yes & Yes & Yes & Yes \\
\hline
\end{tabular}

Both satisfy the first condition of Definition 5.1 for $v, \gamma>1$. The dual-power distortion function has a zero limit as $u \rightarrow 1^{-}$, but does not satisfy the first limit of Condition 2. (here $\left.\lim _{u \rightarrow 0^{+}} g_{D P}^{\prime}(u)=v<\infty\right)$. By contrast $g_{P H}$ has an infinite limit as $u \rightarrow 0^{+}$but does not satisfy the first limit of Condition 2 . $\left(\right.$ as $\left.\lim _{u \rightarrow 1^{-}} g_{P H}^{\prime}(u)=\gamma\right)$. Hence neither is adapted.

By contrast, the WT distortion $g_{\lambda}$ in Eq. 7 is an example of an adapted risk measure (see Wang, 2000 for a derivation of $g_{\lambda}^{\prime}(u)$ at 0 and 1). Table 4 summarizes the conditions met by the common distortion risk measures discussed above.

\section{Conclusion}

This paper does not attempt to solve the problem of inconsistent decisions based on an improper risk measure. Several families of risk measures have been defined in the last few years: convex, coherent, spectral and deviation risk measures, each with its distinctive features. Here we focus on distortion risk measures, as most families above can be generated through a distortion function. This reduces the study of the risk measure behavior to the properties of a distortion function. The contribution here is the definition of two new properties that may help avoid such inconsistent decisions.

Distortion risk measures are defined as the expected value under a transformed distribution, that includes the investor's risk aversion. In other words, different distortion functions re-weigh differently the initial loss distribution. We study here these different re-weightings.

We show that coherent risk measures (with their corresponding concave distortion function) re-weigh large losses with a factor greater than 1, while de-emphasizing small losses.

It is also shown here that coherence is neither sufficient nor possibly necessary to avoid inconsistent decisions, such as those obtained with $V a R_{\alpha}$ (a non-coherent risk measure). For instance, although $C V a R_{\alpha}$ is a coherent distortion risk measure, it still exhibits an inconsistent behavior. The problem is not the lack of differentiability of its distortion function, as Wang (2002) conjectures, but rather its piece-wise linearity.

We propose the completeness and adaptability properties to address these issues. In defining these new properties we ensure that the distortion function not only uses all the information in the original loss distribution, but also that it uses it adequately. This means that the re-weighting generated by the distortion function differs at each loss. We characterize these properties in terms of the derivative of the distortion function. Table 4 summarizes the properties satisfied by several common distortion risk measures. 


\section{References}

Acerbi C (2002) Risk aversion and coherent risk measures: a spectral representation theorem. J Bank Financ 7:1505-1518

Acerbi C, Simonetti P (2002) Portfolio optimization with spectral measures of risk. Working paper, Abaxbank. www.gloriamundi.com

Artzner P, Delbaen F, Eber J-M, Heath D (1997) Thinking coherently. Risk 10:68-71

Artzner P, Delbaen F, Eber J-M, Heath D (1999) Coherent measures of risk. Math Financ 9(3): 203-228

Basak S, Shapiro A (2001) Value-at-risk based risk management: optimal policies and asset prices. Rev Financ Stud 14(2):371-415

Butsic RP (1999) Capital allocation for property-liability insurers: a catastrophe reinsurance application. Casualty Actuar Soc Forum 1-70

Dhaene J, Vanduffel S, Tang Q, Goovaerts M, Kaas R, Vyncke D (2006) Risk measures and comonotonicity: a review. Stoch Models 22:573-606

Dhaene J, Laeven R, Vanduffel S, Darkiewicz G, Goovaerts M (2008) Can a coherent risk measure be too subadditive? J Risk Insur 75:365-386

Delbaen F (2002) Coherent measures of risk on general probability spaces. In: Sandmann K, Schonbucher JP (eds) Advances in finance and stochastics. Springer, Berlin, pp 1-37

Denneberg D (1994) Non-additive measure and integral. Kluwer, Dordrecht

Denuit M, Dhaene J, Goovaerts M, Kaas R, Laeven R (2006) Risk measurement with equivalent utility principles. Stat Decis 24(1):1-25

Duffie D, Pan J (1997) An overview of value at risk. J Deriv 4:7-49

Föllmer H, Shied A (2002) Convex measures of risk and trading constraints. Finance Stoch 6(4): 429-447

Fritelli M, Rosazza GE (2002) Putting order in risk measures. J Bank Financ 26(7):1473-1486

Goovaerts M, Darkiewicz G, Dhaene D (2003a) Coherent distortion risk measure: a pitfall. Working paper presented to 2003 IME conference

Goovaerts M, Kaas R, Dhaene J, Tang Q (2003b) A unified approach to generate risk measures. ASTIN Bull 33(2):173-192

Gzyl H, Mayoral S (2008) On a relationship between distorted and spectral risk measures. Rev Econ Financ 15 (in press)

Kusuoka S (2001) On law invariant coherent risk measure. In: Kusuoka S, Maruyama S (eds) Advances in mathematical economics, vol 3. Springer, Berlin, pp 83-95

Rockafellar RT, Uryasev S (2000) Optimization of conditional value-at-risk. J Risk 2(3):21-41

Rockafellar RT, Uryasev S, Zabarankin M (2006a) Generalized deviation measures in risk analysis. Finance Stoch 10:51-74

Rockafellar RT, Uryasev S, Zabarankin M (2006b) Optimality conditions in portfolio analysis with deviations measures. Math Program Ser B 108:515-540

Ruszczynski A, Shapiro A (2006) Optimization convex risk measures. Math Oper Res 31(3):433-452

Song Y, Yan JA (2006) The representation of two types of functionals on $L^{\infty}(\Omega, \mathcal{F})$ and $L^{\infty}(\Omega, \mathcal{F}, \mathbb{P})$. Sci China Ser A Math 49(10):1376-1382

Venter GG (1991) Premium implications of reinsurance without risk. ASTIN Bull 21(2):223-230

Venter GG (1998) Discussion of implementation of PH-transforms in ratemaking. In: Proceedings of Casualty Actuarial Society, LXXXV, pp 980-989

Wang SS (1996) Premium calculation by transforming the layer premium density. ASTIN Bull 26: $71-92$

Wang SS (2000) A class of distortion operators for financial and insurance risks. J Risk Insur 67:15-36

Wang SS (2002) A risk measure that goes beyond coherence. In: Proceedings of the 2002 AFIR (Actuarial approach to financial risks) Colloquium, Cancun, March 2002

Wirch J, Hardy MR (2000) Ordering of risk measures for capital adequacy. Institute of Insurance and Pension Research, University of Waterloo, Research Report 00-03

Wirch J, Hardy MR (2001) Distortion risk measures: coherence and stochastic dominance. Working paper. http://pascal.iseg.utl.pt/ cemapre/ime2002/ 